\title{
Urgent care out of hours: a comparison of the experiences of older people and parents of young children in a semi-rural area
}

Sue Horrocks and Debra Salmon Faculty of Health and Social Care, University of the West of England, Glenside Campus, Stapleton, Bristol, UK.

\begin{abstract}
Aim: Older people and parents of young children are the most frequent users of out of hours care. However, their needs and expectations of care may be different. The aim of this study was to explore and compare the experiences and views of these two groups following the transfer of responsibility for out of hours services from General Practitioners (GPs) to a Primary Care Trust (PCT). Methods: Qualitative research using semi-structured interviews with 19 informants living in a geographically large, semirural PCT area in England served by 15 GP practices. Interviews were taped, transcribed and analysed using a thematic framework. Findings: Older people presented with more complex health problems than young children, and expressed more reluctance at calling the service. Both groups experienced similar access problems for using the primary care centre (PCC). Older people reported fewer difficulties obtaining a home visit, though experienced continuity problems when illness episodes lasted longer than one shift. Both groups questioned the ability of a doctor to diagnose accurately using only telephone assessment. Conclusion: Despite differences in presenting symptoms and attitudes to service use, older people and parents with young children experienced similar problems in accessing care at the PCC. Older people more frequently received home visits than parents with young children, and it may be that social context is not sufficiently taken into account when assessing need for a home visit. Practitioners should be aware that older people tend to minimise symptoms and should be cautious of relying on lay interpretations of illness when carrying out telephone assessments with this group.
\end{abstract}

Keywords: children; elderly; out of hours care; patient satisfaction; qualitative methods

Received: March 2007; accepted: August 2007

\section{Introduction}

The organisation of urgent care out of hours has been transformed in the last ten years. Historically, General Practitioner (GP) practices provided their own out of hours care supplemented by the use of deputising services and locally organised GP cooperatives, with a further introduction of more

Address for correspondence: Sue Horrocks, Senior Lecturer in Primary Care, Faculty of Health and Social Care, University of the West of England, Glenside Campus, Stapleton, Bristol BS16 1DD, UK. Email: susan.horrocks@uwe.ac.uk premises-based care, where patients could be seen at a primary care centre (PCC) as an alternative to a home visit (Salisbury, 1997a; House of Commons Health Committee, 2004). Government policies to increase access to health services have led to the development of nurse-led walk in centres and National Health Service (NHS) Direct, which can be accessed by patients out of hours (Department of Health, 2000a). Following the new GP contract (Department of Health, 2004a) responsibility for out of hours care provision passed to Primary Care Trusts (PCT), which were enabled to implement the recommendations of an independent review to 
provide an integrated service linking a single call from a patient to the most appropriate service (Department of Health, 2000b). Patient satisfaction with out of hours services was recently evaluated, but pre-dated the transfer of responsibility for out of hours to PCTs and focused on services integrated with NHS Direct (Lattimer et al., 2005). Current quality standards for delivery of out of hours services require local PCTs to audit access and the patient experience (Department of Health, 2004b).

While research on out of hours services has focused on the mode of service delivery (Cragg et al., 1997; Salisbury, 1997b; Lattimer et al., 1998), and factors contributing to patient satisfaction (Salisbury, 1997a; Wilson et al., 2001; McKinley et al., 2002; Leibowitz et al., 2003), the particular perceptions and experiences of older people and parents of young children are comparatively under reported. Responding to the needs and demands of both groups within one service and ensuring equity of access to each may be a difficult balance and insight into the factors that enhance patient satisfaction with services is useful to health planners and providers. Recent findings showed one in five out of hours patients were dissatisfied, suggesting there may be shortcomings in patient experiences currently not captured by PCTs, and a qualitative study may be able to probe the reasons for this more precisely (National Audit Office, 2006).

As yet there are no published studies evaluating out of hours care using data obtained since reconfiguration of services under PCTs. This paper reports the findings of a qualitative study, which was undertaken to explore in more depth the findings of a satisfaction survey (paper in preparation) of views and experiences of older people and parents of young children towards the out of hours care received in the home, by telephone or at a PCC following service reconfiguration. Ethical approval was granted by the local research ethics committee.

\section{Methods}

The study setting comprised a semi-rural community, served by 15 GP practices. During out of hours, face-to-face care and telephone advice up to $11 \mathrm{pm}$ was provided by medical and nursing teams from the PCC at the local general hospital. A control centre provided triage and medial telephone advice from $11 \mathrm{pm}$. A decision support system used by call handlers identified emergency calls. Clinical judgement was used in giving telephone advice. Patients could receive telephone advice, a face-to-face consultation at the PCC up to $11.00 \mathrm{pm}$ or a home visit from a doctor or district nurse.

Participants were selected from a random sample of users who had completed a questionnaire to evaluate their satisfaction with the out of hours service, and who had volunteered to be interviewed about their experiences. Participants were purposively selected, being either a parent of a child or an adult of 60 years or over, having received care across the settings (home visit, PCC or telephone advice), male and female, and according to satisfaction. They were contacted by phone to discuss the study, and a followup letter and information sheet was sent to the home. Participants interviewed at home signed a consent form agreeing to the interview. For those who preferred to be interviewed by phone, consent forms were completed and returned by stamped addressed envelopes.

Semi-structured interviews were carried out by $\mathrm{SH}$ and DS using a topic guide designed to probe in more detail reasons for contacting the out of hours service, hopes and expectations of care, experiences and ideas of how the service could be improved for others (Figure 1). Each interview was carried out in the participant's own home, choice of location or by telephone if this was preferred.

Interviews were carried out one to two weeks following the out of hours consultation and took place between September 2005 and December 2005. These were taped and transcribed verbatim. Participants were invited to view and comment on their transcripts, though all but one felt this to be unnecessarily time consuming. Interviews were analysed by each researcher identifying key concepts and categories, then compared between researchers in order to develop a coding framework based on the original topic guide and issues arising from the participants' experiences. These were then charted to enable comparison between the experiences of older people and parents with young children in different settings. Framework analysis is explicitly geared towards generating policy and practice orientated findings and has been widely used in health related research, as it enables analysis of specific issues that the stakeholders may 
(Exact content and order vary according to responses)

1. Can we start by talking about the reasons why you contacted your doctor out of

surgery hours?

(Probes identified whether the problem was a sudden emergency, or an ongoing

problem, and whether the interviewee was calling on behalf of another)

2. What was your experience in getting help when you called the doctor?

(Probes identified common problems experienced by patients accessing services,

including transport to a health care setting, dependency care issues, access to a

telephone,)

3. What were you hoping would happen when you called your doctor out of surgery

hours?

(Probes assessed whether patient expected to consult a doctor or nurse, and whether

this was an important issue for them)

4. How far were your expectations of the service met by the person who gave you health

care advice or treatment?

(Probes assessed whether patient was content with advice or treatment from health

professional spoken to/seen, and experience of follow up arrangement)

5. How do you think out of hours services could be improved for people in a similar

position to yourself?

(probes identified what measures could address perceived service deficiencies)

Figure 1 Interview topic guide

wish to be addressed (Ritchie and Spencer, 1994). Interviews were coded under the following themes:

Severity of condition, urgency of need for care, hopes and expectations of care, perceptions of access to care, perceptions of quality of care and recommendations for service improvement, and the impact of social context on decision making.

\section{Results}

Three hundred and ten respondents (36\%) volunteered to be interviewed, of whom 88 were parents of children under 13 years and 86 were people 60 years or over.

Ten older people and nine parents of young children were interviewed (Table 1), of these five were by telephone; working parents in particular requested this option. The majority of older participants presented with multiple health problems in addition to the current problem. Most calls for children were for acute, unexplained symptoms associated with pyrexia, giving cause for concern, especially if the child was an infant. Older people more frequently received home visits than young children, who more frequently received care at the PCC. Most care was given by a doctor. Four out of 10 interviews with older people were

Primary Health Care Research \& Development 2007; 8: 367-376 
Table 1 Characteristics of participants

\begin{tabular}{|c|c|c|c|c|c|c|}
\hline No. & $\begin{array}{l}\text { Patient's } \\
\text { age (years) }\end{array}$ & $\begin{array}{l}\text { Patient's } \\
\operatorname{sex}(M / F)\end{array}$ & $\begin{array}{l}\text { Setting } \\
\text { (Home Tel } \\
\text { PCC) }\end{array}$ & Presenting condition & $\begin{array}{l}\text { Practitioner } \\
\text { delivering care } \\
\text { (Dr, Nurse, D. Nurse) }\end{array}$ & Interviewee \\
\hline 1 & 81 & $\mathrm{~F}$ & Home & Cellulitis & $\mathrm{Dr}$ & Patient \\
\hline 2 & 71 & M & PCC & Severe haematuria ${ }^{a}$ & $\mathrm{Dr}$ & Wife of patient \\
\hline 3 & 82 & M & Home & Pain $^{a}$ & Dr & Wife of patient \\
\hline 4 & 92 & M & Home PCC & Acute constipation $^{a}$ & DN Dr & Patient \\
\hline 5 & 60 & $\mathrm{~F}$ & Home & Migraine & $\mathrm{Dr}$ & Patient \\
\hline 6 & 67 & M & Home Tel & $\begin{array}{l}\text { Retention of urine, } \\
\text { haemorrhoids }\end{array}$ & DN Dr & Patient \\
\hline 7 & 65 & $F$ & Home & Back pain, spasm & $\mathrm{Dr}$ & Patient \\
\hline 8 & 81 & M & $\begin{array}{l}\text { Home visit } \\
\text { agreed }\end{array}$ & $\begin{array}{l}\text { Abdominal pain, } \\
\text { vomiting, } \\
\text { haematemesis }\end{array}$ & $\mathrm{Dr}$ & Wife of patient \\
\hline 9 & 82 & $F$ & Tel & Cough & $\mathrm{Dr}$ & Son of patient \\
\hline 10 & 70 & M & Home & Painful ear ${ }^{a}$ & $\mathrm{Dr}$ & Patient \\
\hline 11 & 3 & $\mathrm{~F}$ & Tel & Ankle injury & $\mathrm{Dr}$ & Mother \\
\hline 12 & 3 & $\mathrm{~F}$ & PCC & $\begin{array}{l}\text { Flu, bronchitis and } \\
\text { breathing difficulty }\end{array}$ & $\mathrm{Dr}$ & Mother \\
\hline 13 & 3 days & M & PCC & Infected umbilicus & $\mathrm{Dr}$ & Father \\
\hline 14 & 3 & $\mathrm{~F}$ & Tel & $\begin{array}{l}\text { Temperature, sore } \\
\text { throat, breathing } \\
\text { difficulty }\end{array}$ & $\mathrm{Dr}$ & Father \\
\hline 15 & 7 & $\mathrm{~F}$ & PCC & $\begin{array}{l}\text { Ear \& throat pain, rash } \\
\text { \& facial swelling }\end{array}$ & $\mathrm{Dr}$ & Mother \\
\hline 16 & 7 weeks & $\mathrm{F}$ & Home & Temperature & $\mathrm{Dr}$ & Mother \\
\hline 17 & 3 & M & Tel & Temperature & $\mathrm{Dr}$ & Mother \\
\hline 18 & 6 & $\mathrm{~F}$ & PCC & Temperature & Nurse & Mother \\
\hline 19 & 12 & $\mathrm{M}$ & PCC & $\begin{array}{l}\text { Stomach pain, } \\
\text { appendicitis }\end{array}$ & $\mathrm{Dr}$ & Mother \\
\hline
\end{tabular}

a Patient had multiple health needs.

$\mathrm{PCC}=$ primary care centre.

with their main carers (wives and one son) as the patient was too ill to be interviewed. The majority of interviews with parents were with the mother. Only one parent described calling out of hours for a child with a chronic condition.

To enable ease of comparison of the experiences and perceptions of older participants and parents of young children between settings, findings are reported under the heading of the specific out of hours setting experienced. Some participants experienced more than one setting during the course of the same episode of illness.

\section{Initial contact by telephone}

There were generally no differences in the ease with which initial telephone contacts were made. However, for parents of young children, satisfaction with initial contact by telephone was closely related to the length of time they waited either for their call to be answered or for a doctor to return their call after contact with a call handler. Parents of young children with a high temperature were particularly anxious for an immediate response, as they were concerned about how quickly their children could deteriorate:

Then you start to sort of worrying $20 \mathrm{~min}$ later he's still getting hot, he's 40 [degrees] by then, and I'm starting to think should I ring an ambulance, you know, and I actually started to pack an overnight bag for him, because I was thinking you know, if we've got to go in, to stay in I need to be prepared.

(Participant 17, mother of a three-year-old child with pyrexia)

The clarity of initial advice was vital. Acute anxiety can lead to misunderstanding, frustration 
and panic. A shorthand message as seemingly straightforward as a telephone number can be misinterpreted:

I think I was in an anxious state, I picked up the phone and I was given a telephone number - a recorded message and it was something like two twenty two twenty - I can remember this as if it was ingrained, and I dialled this number as I thought was correct and it was the wrong number and I tried again and it was still the wrong number. So on that recorded message from the surgery there was an NHS direct, so I thought, I will try those. In desperation... "Anybody please!". So I dialled NHS direct. I got through to someone... someone who took all my details, said it would be a recorded message and I didn't mind that at all, and then I was told I would have a phone call back within half an hour...eventually I rang the number again - the doctor's number and then it dawned on me what the correct, what she was trying to tell me - two twenty two twenty - well the two twenty, why didn't she spell it out in numbers?

(Participant 2, husband with haematuria)

\section{Home visits}

Older people received home visits more frequently than young children. Eight older participants had been seen at home either by out of hours doctors or district nurses. Four of the participants lived alone and three did not have easy access to a car. Perceptions of presenting symptoms ranged from painful though not an emergency, to severe pain or vomiting requiring urgent attention. In most cases, older people expressed reluctance to call the doctor, especially at night, despite acute illness:

I was so concerned when he became so distressed... that's why I was prepared to call somebody out, normally I would have said "hey have a couple of paracetamol", but I had already sat with him for an hour and tried to cushion him with pillows and given him some paracetamol and tried to calm him down and it was clearly something much more serious.

(Participant 3, pain, UTI, fall, stroke)
The pain was so bad through the night I just didn't know where to put myself so about 4 o'clock I said I just can't, I just cannot stand it any more, so he (husband) phoned the doctor again about 6am. He said we would wait until 6am because we can't ring in the night, so we phoned about 6am.

(Participant 7, back pain)

Although in contrast to parents of young children, older participants experienced little difficulty in obtaining agreement for a home visit in principle, some reported long delays for home visits, and, where episodes of illness continued over a prolonged period such as a weekend, the continuity of care between visits was questionable:

But the lack of continuity between them is one major issue and I suppose most of the doctors are very, very professional and I would think they would return if necessary. I couldn't think, the third doctor, I didn't know you see, he didn't know about the second doctor, so I said oh has he gone off duty? Maybe that was the reason why he hadn't come back, but he didn't say anything.

$$
\text { (Participant 7, back pain) }
$$

Children tended to present with acute illness, leading to parental anxiety about the possible cause, and potential for rapid deterioration. Some parents reported that they had rung the out of hours wanting a home visit, and received telephone advice or were asked to attend the PCC instead. Parents who did not have their own transport, expressed anxiety about taking a sick or feverish child out at night. If they had other small children they were concerned about getting them out of bed, or finding a sitter at night. Such people may require a home visit because of their practical and social needs, and this is not always taken into account by practitioners. Participant 12, a single parent, described the difficulties:

Well if I'd only have $30 p$ to go up the phone box see, I'd have never ever got through to the doctor, you know. You know, that was my only concern, because I was thinking it's lucky I've got credit on my phone really. Yes, and just the fact that the one time she was really, you know, the first she was about six months old and she had the same thing a 
real bad, but she was very, very breathless, and I said to the doctor I'd really like you to come out because I'm not well myself and my daughter is not very well and I've got no way of getting up to you, I haven't got no travel, and he said, well, get, you know he just really didn't want to come out...

(Participant 12, mother of 3 year old with flu and bronchitis)

Socio-economic or educational differences affect the ways in which patients express their needs and may influence success in obtaining a home visit if required; two older participants emphasised the importance of preparing an effective request for a home visit:

The first one is, what do you want? You've got to impress on them that it's urgent, if it's something that you can wait until the next day they'll tell you.

(Participant 4, constipation)

I think my presentation is such that people would take me seriously if I called a doctor. I wasn't in a panic, I wasn't ... you know I have lived with all the ups and downs for too long to panic any more and I clearly wasn't somebody saying "hey look I have got a nosebleed you have got to come".

(Participant 3, UTI, fall, stroke)

\section{Primary Care Centre}

The PCC was located adjacent to the Accident and Emergency (A\&E) at the local hospital. However, advantages of proximity to the hospital were offset by practical access problems such as distance from home, transport and parking. Despite satisfaction with the quality of care received in this setting, problems due to miscommunication and transfer from car to PCC were common to both user groups resulting in some participants finding it difficult to locate the service at all.

Her (the daughter) took me up the A \& E and they did the usual, so I expected then to be directed to the doctor as she said, come and see me. So they put me on a trolley, ...there was evidently, either the doctor didn't tell reception, or else they were told and they didn't ....so I was up there about three hours and I needn't have been there you know.

\section{(Participant 4 abdominal pain)}

We knew roughly which way we were going, but obviously walking in, it was freezing cold walking with your little one. There was no clear signs, because obviously I was looking for, you know, some sort of big sign like you do in hospital, X-ray sign and stuff like that. And it wasn't. It was a little sign based up in the edge of the corridor and we actually walked, we actually asked quite a few people where it, where it actually was where the ... was.

(Participant 13, father of 3 day old baby with infected umbilicus)

While parents of young children were generally happy with the care they received at the PCC, including wait times, the quality of the care received, assessment, treatment, and the manner and quality of communication of the practitioners, there were areas which some participants felt could be improved. The issue most consistently commented on for improvement was parking and poor signage. Lack of space to park, fear of getting a fine, concerns about distance between parking space and entrance and carrying a sick child all made attendance at the PCC daunting and parents anxious. Parents attending alone also raised the dilemma that they felt a certain amount of emotional conflict about whether to abandon the car or the child on arrival:

... of course I can rush up and I can dump the car somewhere and whiz him in, but I can't then go back and move the car, you know what are you supposed to do? Leave your car somewhere where it's going to get clamped, or be in the way of an ambulance or something, or you know....drive round and round looking for a convenient space. (laugh)

(Participant 17, father of three-year-old child with pyrexia)

you know, if you've got kids and they're poorly and you have to park a long way away from where you need to go in it's just an added stress and complication at a time when actually the family is quite stressed as 
it is. If I've got drag my six year old and say in this instance my six year old and my three year old to get to the hospital it's just more hassle, and so I think they really need to think about it

(Participant 11, mother of three-year-old with ankle injury)

Both older participants and parents of young children were satisfied with the quality of care they received from the PCC. However, both groups identified practical problems with access, such as poor verbal instructions for finding the department, scarce parking, the need to pay for a parking ticket and inadequate signage.

\section{Telephone advice}

Five participants had received telephone advice, and commented on the high level of clinical skills and quality of communication they experienced. Allowing time for parents to discuss their anxieties and the often detailed practical advice given, gave them confidence to manage their child at home without further recourse to the service:

I spoke to a really nice woman GP who talked me through examining E's foot so that I got to, you know, she gave me knowledge that actually would be useful in a similar situation, so she talked to me about trying to find any heat on E's foot, but very calmly talked me through you know what was happening with $\mathrm{E}$ and at the end of it we both ascertained that probably what she'd done was sprained her ankle.

(Participant 11, mother of three-year-old, ankle injury)

However, there was evidence that where a home visit was expected or wanted by the participant, satisfaction with a telephone consultation was significantly reduced.

Then they kept saying well this will do for now and if, if you have any other problems then ring back later, so that was like if we can avoid going this time then if they ring again, that was what I got the feeling..., it was a very horrible feeling really at that time.

(Participant 14, father of three-year-old, sore throat and breathing difficulties)
The complexity of their health problems and tendency to minimise the severity of symptoms experienced by older participants called into question the appropriateness of a telephone assessment. One participant who had called to request a home visit for his mother whose condition seemed to be deteriorating, queried whether it would not have been more appropriate for her to have been seen and examined by a health professional rather than the doctor relying on him as a lay person to assess her condition over the telephone:

It would have improved I think if someone had actually come down and looked at her, and made a judgement, their own judgement, rather than a third party story. I mean I have a personal interest in my mother, so in relating what I see, isn't necessarily what a professional, medical eye would see, or indeed hear.

(Participant 9, cough)

Furthermore patients with a stoical attitude to pain may under report their suffering on the telephone, the signs of which might have been picked up in a face-to-face encounter:

I was feeling in a right state because I couldn't sit down. I just could not sit down... I couldn't sit down for about...(long pause) five days without discomfort you know.

(Participant 6, haemorrhoids)

Although this participant expressed satisfaction with his telephone consultation, the doctor did not visit, advised analgesia (unobtainable at night) and referred him to his own GP the next day, when, on examination, a rectal prolapse was found.

Another participant observed that undue emphasis may have been given to her own assessment that simple constipation might be the cause of her husband's abdominal pain, leading to a low priority allocation for a home visit. After repeated telephone calls to the service with her husband in acute pain and his condition deteriorating and no visit received, his son eventually came from a town forty miles away to take him to hospital where he underwent emergency surgery for a bowel obstruction soon after arrival:

He (the out of hours doctor) only knew that

I had thought he had constipation as far as I 
know, you know. I don't know what else he knew ... They just never came for hours. Whether they thought, well it's only constipation, you know,... I don't know.

(Participant 8, abdominal pain)

While a telephone consultation was valued by some participants who needed reassurance, advice or a practical service such as a prescription, there was evidence from the interviews that where a home visit had been hoped for, but denied, satisfaction was reduced. Some questioned how a doctor could diagnose accurately over the telephone without an examination of the patient, and suggested that undue credence may be given to lay opinion of symptoms, possibly leading to misdiagnosis and potential harm to the patient.

\section{Discussion}

Findings from the interviews confirm patient satisfaction with out of hours care remains high following service reconfiguration, although, as suggested by the Audit Report (National Audit Office, 2006), this masks dissatisfaction which may indicate important quality issues for planners. Despite the dissimilarities in complexity of presenting conditions, and differing social contexts, similar issues and concerns arise for older people and parents of young children. For example, both groups reported practical difficulties in accessing the PCC, and questioned whether telephone assessment can be as reliable as faceto-face examination of the patient. In view of the seriousness of the examples given where telephone assessments might have led to 'missed cases' it is timely to reflect whether the use of decision support software might have prevented the difficulties experienced by some callers.

In this study, parents of young children seemed to find it more difficult to obtain a home visit than older people, which suggests social context, lack of family support, financial resources or access to a transport or telephone may not be taken sufficiently into account when assessing need for a home visit. Older people repeatedly stated great reluctance to call the doctor, particularly at night, and practitioners need to be aware that they tended to endure significant pain and anxiety before making their first call. Older people more frequently needed care for an extended episode of illness and this exposed continuity problems in record keeping and communication between doctors' shifts, with no evidence that subsequent doctors had information relating to an earlier visit. Poor continuity of care may have quality of service implications for patients requiring ongoing care, such as those with chronic conditions or the terminally ill.

Earlier studies undertaken in an urban, socially deprived area using telephone interviews, probed the experiences of people who received telephone advice or visited a PCC before recent changes (Payne et al., 2001; Shipman et al., 2001). The present study in a semi-rural area confirmed the finding that practical barriers to use of the PCC, such as pain and discomfort endured during travel, dependents, transport difficulties (parking and signposting) and expense still exist. Shipman et al. (2001) concluded that those with easy access to resources gained the greatest benefits from out of hours services, being more satisfied with the shorter wait to see a doctor. Inequalities of care evident in a socially deprived area could be wider in a relatively prosperous, rural area, with pockets of disadvantage, though less easy to see. Reduction of these inequalities might be alleviated by the use of patient transport in some circumstances or a protocol to guide practitioners when a home visit might be required on the grounds of social context. In view of the difficulties related to poor signage and parking at the PCC located at the general hospital, there may be implications for current policy if providers are encouraged to develop urgent care centres on hospital sites (DH, 2006).

Surveys have shown that dissatisfaction is associated with unmet expectations (Wilson et al., 2001; McKinley et al., 2002). Interviews suggested that patients who wanted a doctor's visit interpreted the doctor not agreeing to visit as reflecting a lack of sympathy for, or not understanding the seriousness of the problem, a finding reported elsewhere in relation to accessing a GP co-operative (Payne et al., 2001). Our study highlighted different service expectations of the two user groups, with older people coping with significant and painful symptoms for some time yet calling the doctor only with significant reluctance, a stoical attitude to illness reported elsewhere (Foster et al., 2001).

The study was carried out in one PCT area with a relatively affluent population living in a largely 
rural area with a higher than average number of older people. Interviews, with a volunteer purposive sample of users, were carried out as soon as possible following the out of hours contact to reduce the effect of recall bias. These were carried out in the participant's home where preferred, in order not to exclude frail or disabled older people who may not be able to use the telephone effectively, but may not reflect the views of questionnaire non-responders or those with for whom English is not their first language. The study took place during the autumn and winter months and difficulties of locating the PCC and in taking sick children out of doors at night and in cold weather may have been a particular concern of users in these circumstances. Nevertheless the study took place in a comparatively large PCT area, where services were organised using a typical configuration of services. It highlighted and confirmed that although older people and young children are dissimilar in presentation of symptoms, they may share common problems in accessing the out of hours service.

\section{Conclusion}

Organisational changes resulting in the transference of responsibility for out of hours care to PCTs and a reconfiguration of services to enable a single point of contact have not yet removed some of the practical barriers to accessing services. Generally high levels of satisfaction mask problems encountered by older people and parents of young children, such as difficulty in accessing a home visit and poor continuity of care. Telephone advice continued to be a source of dissatisfaction for some users, with participants of this study questioning the ability of a doctor to carry out a thorough assessment without a face-to-face encounter.

\section{Acknowledgements}

The study team would like to thank all the people who gave their time to be interviewed and Jane Brown, Project Nurse, and Emma Griffin, Research Secretary for co-ordinating the study. Funding was provided by Cheltenham PCT and The University of the West of England, Bristol. Ethics committee approval was granted by Gloucestershire Research Ethics Committee.

\section{References}

Cragg, D.K., McKinley, R.K., Roland M.O., et al. 1997: Comparison of out of hours care provided by patients' own general practitioners and commercial deputising services: a randomised controlled trial: I. The process of care. BMJ $314,187-89$.

Department of Health. 2000a: The NHS plan: a plan for investment a plan for reform. London: Department of Health.

Department of Health. 2000b: Raising standards for patients: new partnerships in out of hours care. An independent review of GP out of hours services in England. London: The Stationary Office.

Department of Health. 2004a: Statutory Instrument No. 291 The National Health Services (General Medical Services Contracts) Regulations. London: HMSO http://www. legislation.hmso.gov.uk/si/si2004/20040291.htm.

Department of Health. 2004b: National quality requirements in the delivery of out of hours services. London: Department of Health.

Department of Health. 2006: Direction of travel for urgent care: a discussion document. London: Department of Health.

Foster, J., Dale, J. and Jessopp, L. 2001: A qualitative study of older people's views of out of hours services. British Journal of General Practice 51, 719-23.

House of Commons Health Committee. 2004a: GP out of hours services 5th report of session 2003-4, Volume 1. London: The Stationary Office.

Lattimer, V., et al. 2005. Evaluation of the exemplar programme for integrated out of hours care: final report. University of Southampton. Retrieved 2 February 2006 from http://www.nursingandmidwifery.soton.ac.uk/exemplar/ index.htm.

Lattimer, V., George, S. Thompson, F., et al. 1998: Safety and effectiveness of nurse telephone consultation in out of hours primary care: randomised controlled trial. BMJ 317, 1054-059.

Leibowitz, R., Day, S. and Dunt, D. 2003: A systematic review of different models of after hours primary medical care services, and patient and GP satisfaction. Family Practice 20, 311-17.

McKinley, RK., Stevenson, K., Adams, S. and Manku-Scott, TK. 2002: Meeting patient expectations of care: the major determinant of satisfaction with out-of-hours primary medical care. Family Practice 19, 333-38.

National Audit Office. 2006: The provision of out-of-hours care in England: report by the Comptroller and Auditor General. London: National Audit Office.

Payne, F., Shipman, C. and Dale, J. 2001: Patient's experiences of receiving telephone advice from a GP co-operative. Family Practice 18, 156-60.

Ritchie J. and Spencer L. 1994: Qualitative data analysis for applied policy research. In Bryman, A. and Burgess, R.G., editors, Analysing qualitative data. London: Routledge. 
Salisbury, C. 1997a: Postal survey of patients' satisfaction with a general practice co-operative. BMJ 314, 1594-598.

Salisbury C. 1997b: Observational study of a general practice out of hours cooperative: measures of activity. BMJ. Retrieved 17 June 2004 from http://bmjjournals.com/cgi/ content/full/314/7075/182.
Shipman, C., Payne, F., Dale, J. and Jessopp, L. 2001: Patientperceived benefits of and barriers to using out of hours primary care centres. Family Practice 18, 149-55.

Wilson, P., McConnachie, A., O'Donnell, C.A., Ross, S., Moffatm, K.J. and Drummond, N. 2001: Assessing dissatisfaction with an out of hours service: reasons and remedies. Health Bulletin 59, 37-44. 